\title{
Three-dimensional kinetic Monte Carlo simulation of crystal growth from solution.
}

\author{
S. Piana and J.D. Gale* \\ Nanochemistry Research Institute, Department of Applied Chemistry, Curtin University of Technology, \\ PO Box U1987, Perth 6845, Western Australia
}

\begin{abstract}
The growth of urea crystals from water and methanol solutions has been studied with kinetic Monte Carlo simulations. Parameters for the simulations were derived from atomistic molecular dynamics simulations of the growth and dissolution of urea from water and methanol solutions. This approach allows the effect of solvation on the growth and dissolution kinetics to be fully included while extending the size of the simulation to the micrometre length scale and millisecond timescale. .
\end{abstract}

PACS2003: 31.15.Qg, 31.70.Ks, 81.10.Dn

Keywords: A1 nucleation, A2 growth from solutions, A1 computer simulation, B1 Organic compounds

*Corresponding author, Phone: +61 89266 3838, e-mail: J.Gale@curtin.edu.au 


\section{Introduction}

The growth of organic crystals from saturated solution is an important chemical process, and plays a key role in the purification of pharmaceuticals, for instance. The growth of polar organic molecular crystals is often dominated by interaction with the solvent. For this reason, the choice of the solvent and of the crystallization conditions can determine not only the rate of crystallization, but also the crystal morphology or even the particular polymorph that is obtained. Clearly, it would be highly desirable to be able understand in detail the factors that influence the growth of an organic crystal from different solution conditions. Unfortunately, this type of fundamental understanding is largely missing as the interplay between solvent-solute, surface-solvent and surface-solute interactions is very subtle and cannot be easily modelled with methods based on a static representation of the crystal surface and its environment.

Molecular dynamics (MD) simulations offer an attractive alternative way to study the growth of molecular crystals from solution since they are not limited to a single structure, but make it feasible to calculate the properties of an ensemble of conformations therefore fully incorporating entropic contributions. Furthermore, this approach allows the time evolution of the system to be followed and, in favourable cases, permits the direct observation of the processes of interest at the atomic level of resolution. Although appealing, the direct simulation of the growth of a crystal with molecular dynamics techniques is impractical. This type of approach is typically limited to the simulation of a few thousand atoms for a few nanoseconds of real time. Even the smallest and fastest growing molecular crystals are larger than this size and grow on much longer time scales. However, if the global process of crystal growth is considered as the sum of a large number of almost independent processes that occur at the molecular level, it is still possible to use MD simulations to extract information about the molecular level of crystal growth and then use this information as the basis for performing simulations with other methodologies that allow the exploration of larger sizes and greater time lengths.

Here we use MD simulations to characterize the crystal-solvent interface of a molecular crystal (urea) in two different solvents (water and methanol) at the atomic level of resolution. The information obtained from these simulations is subsequently used to extend the simulation of crystal growth to the micrometre size and millisecond timescale ${ }^{1 ; 2}$. These simulations show that with this methodology it is possible to capture the difference between the growth kinetics from water and methanol, and to explore the role of extended defects, such as screw dislocations, on the growth rates. Furthermore, without any fitting to experimental thermodynamic or kinetic data for the crystal growth process, this method allows the reproduction of thermodynamic, as well as kinetic, effects, such as the dependence on the crystal size of growth rates and supersaturation. 


\section{Methods}

Molecular dynamics simulations. The initial coordinates for the simulations were generated with the program GDIS $^{3}$ from the unit cell determined by X-ray diffraction ${ }^{4}$. The surface model was composed of $8 \times 8,6 \times 6$ and $8 \times 8$ unit cells for the [001], [110] and [111] surfaces, respectively. The depths of the urea slabs were 6,8 and 5 unit cells, respectively. The 2-D cells were converted into 3 -D cells with the $c$-axis perpendicular to the surface and a magnitude $25 \AA$ larger than the thickness of the urea slab. The gap between the two surfaces was filled with solvent molecules using the genbox program ${ }^{5}$. The final systems consisted of 768, 576 and 320 urea molecules and 1295, 931 and 631 solvent molecules (water or methanol). The SPC water model ${ }^{6}$ was used in conjunction with the urea potential originally developed by Duffy et al. ${ }^{7}$ and subsequently modified $^{8}$. All the simulations were run with the program GROMACS ${ }^{5}$. The Particle Mesh Ewald ${ }^{9}$ method was used for long-range electrostatic with a short-range cutoff of $0.9 \mathrm{~nm}$. The timestep for the MD simulation was 2.0 fs. NPT molecular dynamics simulations were performed by coupling the system to a Nosé-Hoover thermostat ${ }^{10 ; 11}$ and to a Berendsen barostat ${ }^{12}$ with relaxation times of 4.0 and $5.0 \mathrm{ps}$, respectively. An anisotropic pressure coupling was used with three independent barostats for the $\mathrm{x}, \mathrm{y}$ and $\mathrm{z}$ directions. Solvent molecule positions were first relaxed by geometry optimization and then the density of the system was equilibrated by performing 300 ps of MD simulation at $300 \mathrm{~K}$ with a variable cell along the $c$-axis and the urea molecules fixed. The whole system was equilibrated by performing 200 ps of NPT MD simulation at $150 \mathrm{~K}$ followed by 800 ps of MD simulation at $300 \mathrm{~K}$. After equilibration, six MD simulations of 12, 60 and $12 \mathrm{~ns}$ of MD simulations were performed for surfaces [001], [110] and [111], respectively, and the trajectories analyzed to calculate reaction rates.

System partitioning: In order to calculate reaction rates, the trajectories were subdivided into 50 ps intervals and in each interval the urea molecules were partitioned into crystal-like and solution-like states. A crystal-like urea molecule is defined as a molecule whose average dipole moment orientation (during the $50 \mathrm{ps}$ interval) is within $20^{\circ}$ of the crystal $c$-axis and has a standard deviation from the average orientation of less than $10^{\circ}$. This scheme allows the selection of all the urea molecules that have the correct orientation and are not free to rotate. Other type definitions, based on alternative criteria, are indeed possible, but are expected to give similar results. The robustness of this definition with respect to the sampling interval (50-100 ps), the tolerance on the orientation $\left(10-20^{\circ}\right)$ and standard deviation $\left(10-20^{\circ}\right)$ has been tested. It turns out that the definition is robust, as changes in the parameters within these ranges select essentially the same crystal molecules. 
Crystal molecules were further partitioned in 36 subclasses according to a nearest neighbor representation. Given the $P-42{ }_{1} m$ symmetry of the urea lattice ${ }^{13}$, each urea molecule in the crystal is in contact with four non-equivalent other urea molecules. A classification based on the number of nearest neighbors leads to a total of 36 non-equivalent crystal types ( 1 bulk crystal type, 10 types of kinks, 8 types of steps, 4 types of terraces and 12 types of molecules with one or two neighbors only). Crystal molecules with no neighbors were assigned to the solution-like type.

Calculation of dissolution and crystallization rates. Dissolution and crystallization rates $\left(\mathrm{s}^{-1}\right)$ for each surface site were calculated from the MD simulations in water and methanol at intervals of 50 ps by counting the number of molecules that have moved from a crystal site to the solution, and vice versa, in subsequent time intervals. The numbers of events were then divided by the site and solution concentrations to obtain reaction rates. For some of the rates, multiple values are reported based on the same process occurring at different surfaces. It was found that for certain surface types the nearest neighbor approximation was not sufficient to discriminate between sites that had markedly different rates. In these cases, a pseudo-second nearest neighbor approximation was used instead. Each of these types was divided into three and different rates were calculated for molecules i) with the largest number of second nearest neighbors on the $Z$ direction; ii) with the same number of second nearest neighbors in the $\mathrm{Z}$ and the $\mathrm{X}$ or $\mathrm{Y}$ directions; iii) with the largest number of second nearest neighbors in the $\mathrm{X}$ or $\mathrm{Y}$ direction.

Kinetic Monte Carlo simulations. Simulations were performed with software developed by us specifically for the present study. The program has been highly optimized in order to perform memory and cpu efficient two and three-dimensional kinetic Monte Carlo (KMC) simulations of the growth and dissolution of urea based on the probabilities to observe a reactive event during a time interval of 50 ps calculated from the molecular dynamics simulations. At every Kinetic Monte Carlo step, each surface site (occupied or unoccupied) was evolved according to the probability of observing a reactive event for that site in the time interval $\delta t$, where $\delta t=50 \mathrm{ps}$. After all the surface sites have been tested, the clock is advanced by 50 ps and the process is repeated. This scheme can run on parallel architectures with a very high efficiency and makes it feasible to perform simulations on systems composed of $\sim 10^{9}$ particles for several microseconds.

The major difference between the KMC scheme used in this work and from the approach adopted in ref. ${ }^{1}$ is that, due to computational efficiency, in the present work only two types of urea molecules were considered: crystal-like and solution-like. Urea molecules adsorbed on the crystal surface were considered as solution-like. Comparative calculations between the two approaches show that for urea both schemes yield very similar results. 
Starting conformations for the two dimensional KMC simulations were defect-free crystal surfaces, while those for the three dimensional case were tetragonal particles. In the first steps of the simulation the morphology of the crystal evolves to the equilibrium shape and therefore the arbitrary choice of initial configuration subsequently becomes irrelevant. This shape is then retained during the remaining crystal growth process.

\section{Results}

Classical molecular dynamics simulations.

Molecular dynamics simulations of the [001], [110] and [111] crystal faces in water and methanol were carried out ${ }^{2}$. The simulations were started with a clean crystal face in contact with a pure aqueous solution. During the simulation, the urea crystal dissolves until the saturated solution concentration is reached. At this point, local crystallization and dissolution events are observed, but the average concentration of the solution does not change ${ }^{1}$. More details about these simulations can be found elsewhere ${ }^{1 ; 2}$. Here we will focus on an energy analysis of the crystallization-dissolution reaction. A snapshot of the simulation of the [110] face in methanol is shown in Figure 1.

If an anisotropic pressure coupling is used, after a few nanoseconds of MD simulation a distortion of the crystal lattice is observed and the $a$ and $b$ cell axes are no longer equivalent since $a$ increases by $15 \%$ and $b$ decreases by $13 \%$ (Figure 1 ). An energetic analysis reveals that the distorted crystal structure is lower in energy with respect to the regular high symmetry one. The distortion is due to a slight overestimation of the dispersion interaction between urea molecules with respect to the electrostatic interaction in the urea potential used for the simulations. This is not completely unexpected as this potential has been created to accurately reproduce the urea molecule in solution $^{7 ; 8}$ and has not been fitted at all on any crystal lattice data. However, in this work we are focusing on the thermodynamic properties of the process of dissolution and crystallization of the urea crystal. The agreement of thermodynamic properties with experiment is remarkable. The calculated sublimation energy is $92.4 \mathrm{~kJ} \mathrm{~mol}^{-1}$, which is almost within $1 \mathrm{~kJ} \mathrm{~mol}^{-1}$ of the experimental value, $93.5 \mathrm{~kJ} \mathrm{~mol}^{-1}{ }^{14}$. The dissolution enthalpy can be obtained from the slope of the total energy with respect to the number of dissolved urea molecules and is $13.7 \mathrm{~kJ} \mathrm{~mol}^{-1}$ in water (experiment $13.8 \mathrm{~kJ} \mathrm{~mol}^{-1}{ }^{15}$ ) and $9.7 \mathrm{~kJ} \mathrm{~mol}^{-1}$ in methanol (no experimental value is currently available). The saturated solution concentration at $298 \mathrm{~K}$ was estimated as the concentration at which the crystal neither grows nor dissolves in the MD simulations. Values ranging between 7 and $11 \mathrm{M}$ have been calculated in water (experiment $11 \mathrm{M}^{16}$ ). In methanol, only the simulation of the [001] face reached equilibrium. The equilibrium concentration of the solution in this simulation was 6.5 $\mathrm{M}$ (experiment $4 \mathrm{M}^{17}$ ). The fact that both the enthalpy and the saturated solution concentration 
are correctly reproduced indicates that the entropy contribution to the dissolution is also correct. We conclude that this potential model is less than perfect for the calculation of the structural or mechanical properties of urea, such as the elastic constants. However, the calculated lattice energy is correct and the force field yields a good description of the thermodynamics of the crystallization from solution.

In the present work, the assumption is made that the properties determined from the molecular dynamics simulations are independent of the solution concentration. Although this is not strictly correct, the error due to assuming ideal behaviour is expected to be less than $2 \mathrm{~kJ} \mathrm{~mol}^{-1}{ }^{8}$, which is less than the statistical error. Indeed, plots of the dissolution enthalpy as a function of the number of molecules in solution have been found to be essentially linear, both in water and methanol.

The equilibrium phase of the simulations can be used to study the structure of the crystal surface in contact with a saturated solution. The radial distribution functions calculated for the [001] and [110] faces in water and methanol show that there is a double layer of adsorbed urea molecules on the two crystal faces, in agreement with previous simulations ${ }^{18}$. In an early work by Liu et al. ${ }^{19}$ the aspect ratio of urea crystals grown from water solution was related to the fraction of effective growth units present on the surface, where a growth unit was defined as a urea molecule adsorbed on the crystal surface having a crystal-like orientation and coordination. The free energies of adsorption of a urea molecule on the [001] and [110] surfaces, as well as those of reorientation, and of incorporation of a molecule into a kink, have all been calculated from the MD rates and are reported in Table 1.

The results obtained for the growth on the [001] surface from water and methanol are remarkably similar, suggesting that the two faces should grow at a similar rate, with the growth from methanol being slightly faster than that from water. On the other hand, the energy of incorporation at a kink site on the [110] face in water is very different from that in methanol. While in methanol this energy is negative, in water it is positive. This observation suggests that very different growth rates, and therefore different aspect ratios, should be observed for the [110] face in the two solvents. This is in complete agreement with the morphologies observed experimentally, where the $[001] /[110]$ aspect ratio of urea crystals grown from methanol can be orders of magnitude smaller ${ }^{20}$ than the aspect ratio of crystals grown from aqueous solutions ${ }^{21}$.

Crystallization and dissolution reaction rates. The 3-step partitioning scheme proposed in Table 1 already offers a qualitative understanding of the kinetic step that is primarily responsible for the different aspect ratios observed in crystals grown from aqueous and methanol solutions. However, such a simplified scheme does not capture the fine details of the growth mechanism. For example, it does not explain the polar morphology observed experimentally ${ }^{20}$ or predict the growth mechanism 
on the different crystal faces. This is still possible, but a more sophisticated scheme has to be used. Each MD simulation trajectory was subdivided in $50 \mathrm{ps}$ intervals and within each interval urea molecules were classified as either solution-like or crystal-like. Surface urea molecules are the crystal-like molecules that have less than six nearest neighbors. Surface urea molecules are then further subdivided in types according to the number and position of the nearest neighbors (Figure 2).

We therefore assign to each surface molecule a label that indicates which types of neighbors it is in contact with. For example, a molecule with two neighbors, one of type A and one of type 1, is classified as A1. According to our definition, the crystal types range from 1 (molecule with a single neighbor of type 1) to AB2211 (a crystal molecule with 6 neighbors). To give an example, there are two possible types of kink molecules (A21 and B21) and four possible types of terrace molecules (AB221, AB211, A2211 and B2211). The same partitioning scheme is applied to the vacant sites on the crystal surface. A dissolution event for a certain surface type is counted every time that a urea molecule of that type changes its status from crystal-like to solution-like in subsequent time intervals. A crystallization event is counted every time the reverse process is observed. Reaction rates for each site are calculated as the number of events divided by the total time of the simulation and the average concentration of species ${ }^{1}$. Activation energies can be calculated from the reaction rates assuming that the rate for a barrierless process is equal to the diffusion limit. Relative energies for each surface site can be calculated from the crystallization and dissolution rates obtained from the MD simulations in water and methanol (Figure 3).

Although there are large variations between different sites, the overall free energies calculated in water and methanol are remarkably similar. Nevertheless, very different crystal morphologies and growth mechanisms are obtained from KMC simulations ${ }^{2}$. This observation highlights the importance of having a method that accurately reproduces all energy contributions to the growth process, as small variations in the relative energy of growth from a certain site can lead to large changes in the predicted morphology and growth mechanism.

Kinetic Monte Carlo simulations of crystal growth. KMC simulations were performed on twodimensional and three-dimensional systems to investigate the influence of the extended defects and crystal size on the growth rate. As a first step, the concentration of the saturated solution $C^{s}$ in the KMC experiments was determined. $C^{s}$ was defined as the solution concentration at which a [110] surface with a screw dislocation on it neither grows nor dissolves, and it is calculated to be $5 \mathrm{M}$ and $4 \mathrm{M}$ for water and methanol, respectively ${ }^{2}$. The supersaturation $C^{*}$ is defined as $\left(C-C^{s}\right) / C^{s}$. 
The effect of a screw dislocation on the growth rates of the [001] and [110] faces from methanol at a supersaturation of 0.08 was calculated by performing $2 \mathrm{D}$ simulations of the growth of a $0.1 \times 0.1$ $\mu \mathrm{m}$ surface (Figure 4)

It turns out that the screw dislocation enhances the rate of growth of the [110] face by $\sim 80 \%$, while the effect on the [001] face is less than 5\%. This is consistent with the observation that at this supersaturation the growth on the [110] face is limited by nucleation, while on the [001] surface it is not $^{2}$. In a three-dimensional crystal growing at low supersaturation the overall aspect ratio is expected to be strongly influenced by the presence of such a defect since it selectively enhances the rate of growth on a specific face. From the KMC simulation performed on the [110] face it is immediately apparent that the rate of step propagation in the $+c$ and $-c$ directions is different (Figure 4). This observation is again consistent with the results of three-dimensional simulations of crystal growth from methanol ${ }^{2}$. Indeed, due to the crystal symmetry, on the same face the two directions are not equivalent. This asymmetry is captured by the molecular dynamics simulations, but is completely neglected in the simplified representation of Table 1. It can be satisfactorily reproduced only when the more sophisticated surface type partitioning based on the next-nearest neighbor types is used in the $\mathrm{KMC}$ simulations.

Three-dimensional KMC simulations of growth from methanol solutions were performed with starting nuclei of different sizes and the concentration of the solution at which the nucleus neither grows nor dissolves has been measured. These simulations were performed with a fixed number of urea molecules in the solution. As a result, the solution concentration in the KMC simulation was changing as the crystal was growing or dissolving. This computational setup makes it possible to determine the saturated solution concentration for a nucleus of arbitrary size; as the original nucleus evolves, the concentration of the solution reacts to the changes until a stationary state is reached where no net growth or dissolution is observed. The solution concentration at the stationary state is the saturated solution concentration for that given size. The results clearly show that there is a strong dependence of the saturated solution concentration on the size of the crystal nucleus when this approaches the nm range (Figure 5).

It might appear somewhat surprising that this effect, which is ultimately dictated by the thermodynamic stability of the crystal nucleus, can be captured by the approach proposed here that is purely based on the growth kinetics. However, it should be recalled that the energy of each crystal site can be directly determined by the logarithm of the ratios of growth and dissolution. Therefore, the very same rates also determine the overall stability of a crystal particle in the limit that it can be described as the sum of the contributions of the stability of the molecules that compose it. 
The dependence of the growth rate at the [001] and [110] faces with respect to the surface size was determined. KMC simulations of growth from methanol at a supersaturation of 0.07 were performed. Square two-dimensional [001] and [110] faces of sizes ranging between $6.2510^{-4}$ and $0.64 \mu^{2}$ were simulated. It turns out that growth on the [001] faces is dependent on the surface area only up to a surface size of $0.04 \mu \mathrm{m}^{2}$. On the other hand, at this supersaturation growth on the [110] face is strongly dependent on the surface area up to a surface size of $0.5 \mu \mathrm{m}^{2}$, with growth on the largest surface investigated being 50 times faster than growth on the smaller surface. This effect is related to the relative rates of nucleation and growth on a crystal face. If a birth-and-spread mechanism is assumed, two limiting cases are possible: i) nucleation is so slow that the time required to grow the new layer once a critical $2 \mathrm{D}$ nucleus is formed is negligible; ii) growth is so slow that the time required to create a nucleus is negligible. A real system will be somewhat intermediate between these two extreme cases; for urea at a supersaturation of 0.07 the [110] face is closer to case i) and the [001] surface is closer to case ii). However, the rate of nucleation is proportional to the surface area, while the time required to grow a nucleus to achieve complete surface coverage is inversely proportional to the square root of the surface area. Therefore, if the surface area increases, the rate of nucleation increases, while the rate of spreading decreases. Eventually, a balance between the two contributions will be reached. At this stage, the growth rate becomes constant as any increase in the surface will increase the probability to grow multiple nuclei simultaneously and the average area covered by each nucleus will remain constant. It is important to note that for some crystal faces, in particular at low supersaturation, this limit might never be reached. We suggest that this effect sometimes might contribute significantly to the wide distribution of sizes and shapes observed in crystallization experiments.

\section{Conclusions}

A method based on atomistic MD simulations to perform two- and three-dimensional KMC simulations of the growth of organic crystals from solution has been introduced. Here we have show how these simulations can be used to obtain insights into the kinetics and thermodynamics of the growth of real crystals. In particular, the influence of size and of defects on the observed growth rates has been discussed and related to the growth mechanism.

The method has been successfully applied to urea, but it is completely general and can, in principle, be applied to the study of the growth of any crystal from any solution. The principal limitation to the range of applicability is determined by the rate of the molecular crystallization and dissolution steps. In order to calculate a reliable rate for a reactive event, this event should occur at least a few times during the MD simulation. The typical times currently accessible to a single MD simulation 
are $\sim 10^{-7} \mathrm{~s}$. This might prevent the observation of the some of the slowest steps. However, the basic assumption of this work is that individual molecular steps are independent of each other. The direct consequence of this assumption is that it is possible to combine the results of multiple independent MD simulations to calculate the reaction rates. Therefore, it is possible to take full advantage of parallel or distributed supercomputing to greatly extend the total simulation time and the range of applicability of this methodology.

\section{Acknowledgements}

We would like to thank the Government of Western Australia for their support through the Premier's Research Fellowship program, as well as funding from the Australian Research Council for a fellowship for SP. The provision of computing time by APAC and IVEC is also gratefully acknowledged.

\section{References}

(1) Piana, S.; Gale, J. J Am Chem Soc 2005, 127, 1975-1982.

(2) Piana, S.; Reyhani, M.; Gale, J. D. Nature 2005, 438, 70-73.

(3) Fleming, S D and Rohl, A L. Z Krist . 2005, 220, 1-5.

(4) Swaminathan, S.; Craven, B. M.; Spackman, M. A.; Stewart, R. F. Acta Crystallogr 1984, B40, 398-404.

(5) Lindhal, E.; Hess, B.; van der Spoel, D. J Mol Mod 2001, 7 , 306-317.

(6) Berendsen, H. J. C.; Postma, J. P. M.; van Gunsteren, W. F.; Hermans, J. Intermolecular forces; Reidel: Dordrecht, The Netherlands, 1981; pp 331-342.

(7) Duffy, E. M.; Severance, D. L.; Jorgensen, W. L. Israel J of Chem 1993, 33, 323-330.

(8) Smith, L. J.; Berendsen, H. J. C.; van Gunsteren, W. F. J Phys Chem B 2004, 1065-1071.

(9) Essman, U.; Perera, L.; Berkowitz, M. L.; Darden, T. A.; Lee, H.; Pedersen, L. G. J Chem Phys 1995, 103, 8577-8593.

(10) Nosé, S. Mol Phys 1984, 52, 255-268.

(11) Hoover, W. G. Phys Rev A 1985, 31, 1695-1697.

(12) Berendsen, H. J. C.; Postma, J. P. M.; van Gunsteren, W. F.; DiNola, A.; Haak, J. R. J Chem Phys 1984, 81, 3684-3690.

(13) Swaminathan, S.; Craven, B. M.; McMullan, R. K. Acta Cryst 1984, B40, 300-306.

(14) Ferro, D.; Barone, G.; Della Gatta, G.; Piacente, V. J Chem Thermodyn 1987, 19, 915-923. 
(15) Pickering, M. J Chem Educ 1987, 64, 723-724.

(16) Pinck, L. A.; Kelly, M. A. J Am Chem Soc 1925, 47, 2170-2172.

(17) Boomadevi, S.; Dhanasekaran, R.; Ramasamy, P. Crys Res Technol 2002, 37, 159-168.

(18) Boek, E. S.; Briels, W. J.; Feil, D. J Phys Chem 1994, 98 , 1674-1681.

(19) Liu, X. Y.; Boek, E. S.; Briels, W. J.; Bennema, P. Nature 1995, 374, 342-345.

(20) Docherty, R.; Roberts, K. J.; Saunders, V.; Black, S.; Davey, R. J. Faraday Discuss 1993, 95, 11-25.

(21) Davey, R.; Fila, W.; Garside, J. J Cryst Growth 1986, 79, 607-613. 
Table 1. Free energies $\left(\mathrm{kJ} \mathrm{mol}^{-1}\right)$ of key steps in the crystallization process for the [001] and [110] faces calculated from the MD simulation reaction rates. In this simplified scheme, the process is considered to occur in three sequential steps: i) adsorption of a molecule on a flat surface, ii) reorientation of the molecule to lie in phase with the crystal lattice and iii) incorporation of the molecule at a kink site. Barriers and relative energies are calculated with respect to the previous step.

\begin{tabular}{|l|r|r|r|r|r|c|}
\hline & \multicolumn{3}{|c|}{$[001]$} & \multicolumn{3}{c|}{$[110]$} \\
\hline Water & Adsorption & Reorientation & Incorporation & Adsorption & Reorientation & Incorporation \\
\hline Barrier & 11.5 & 16.1 & 5.07 & 2.9 & 20.2 & 10.8 \\
\hline Stability & 9.5 & 3.9 & -4.0 & 0.3 & 8.4 & 5.8 \\
\hline Methanol & & & & & & \\
\hline Barrier & 8.8 & 14.2 & 4.9 & 4.6 & 16.0 & 10.3 \\
\hline Stability & 7.4 & 1.8 & -4.0 & 0.9 & 6.2 & -3.9 \\
\hline
\end{tabular}


Figure 1. Snapshot from the molecular dynamics simulation of the [110] face in methanol. Urea atoms are colored in blue (nitrogen), red (oxygen) and cyan (carbon). Methanol molecules are colored in green. Hydrogen atoms are not shown for clarity. The lattice distortion induced by the anisotropic pressure scaling is clearly visible in this orientation.

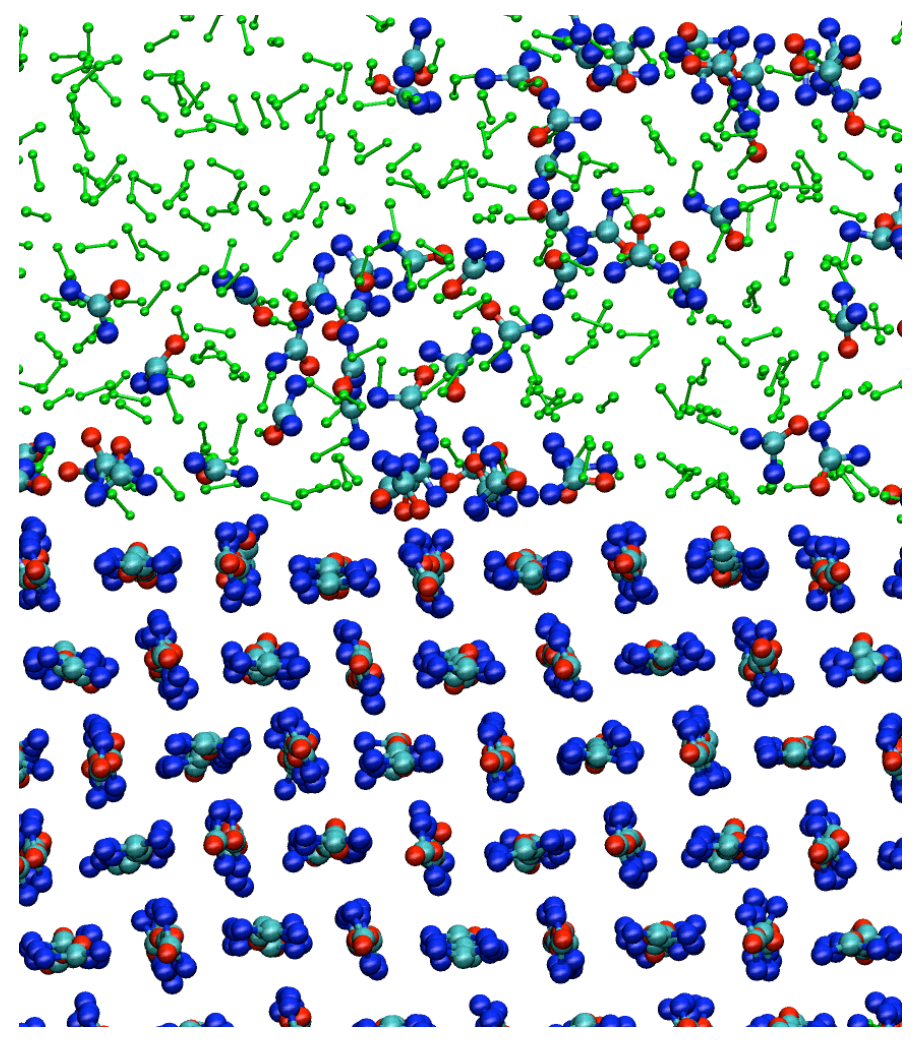


Figure 2. Nearest neighbours of each urea molecule in the crystal lattice. Molecules that are directly hydrogen bonded to the central molecule are drawn in a ball and stick representation. Symmetry equivalent nearest neighbours are represented with the same colour. Note that there are two symmetry-related urea molecules in the unit cell.

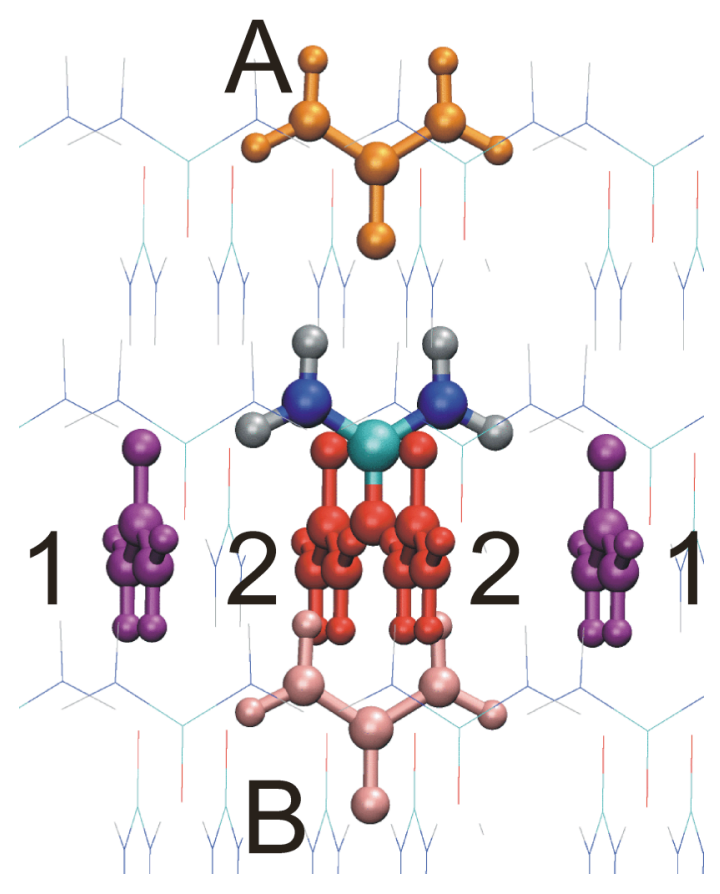


Figure 3. Scatter plot of the surface site free energies calculated in water and methanol. While variations of up to $35 \mathrm{~kJ} \mathrm{~mol}^{-1}$ are observed for energies calculated for different sites, the average deviation between values calculated for the same site in the two solvents is only $2.4 \mathrm{~kJ} \mathrm{~mol}^{-1}$. The dotted line represents the line of linear correlation, with slope 1.0, as a guide to the eye.

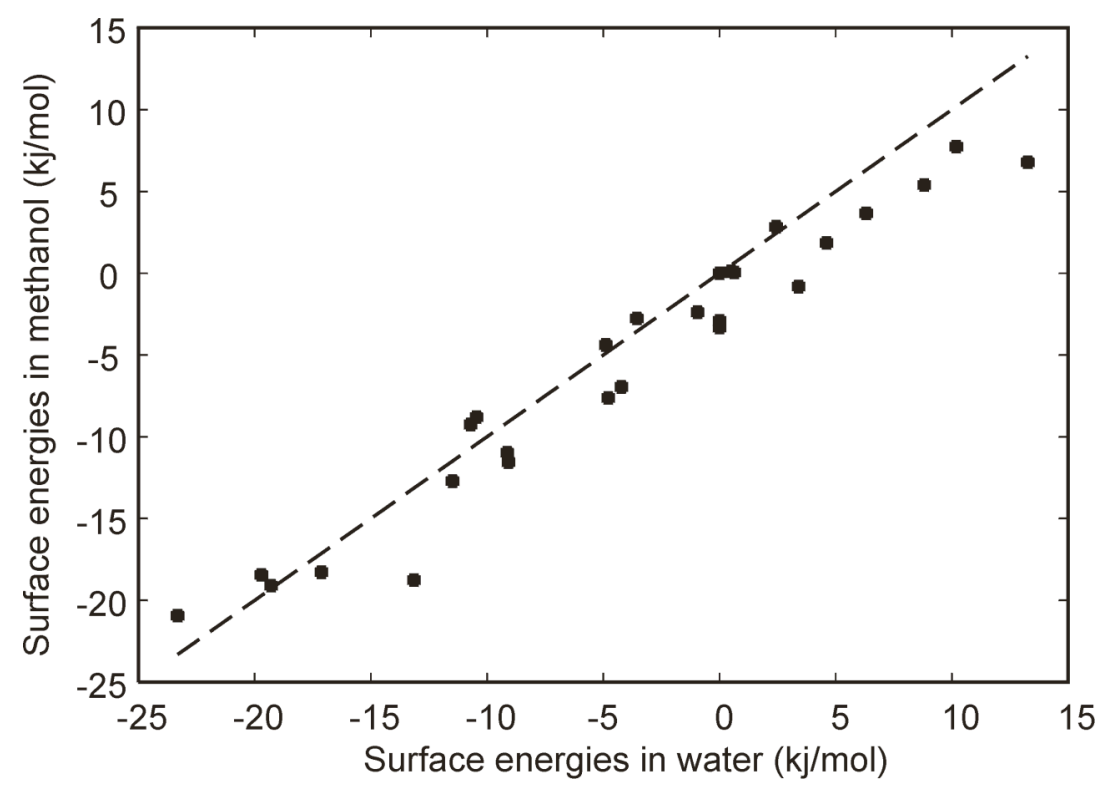


Figure 4. Two-dimensional KMC simulation of growth on the [110] face in the presence of a screw dislocation. The position of the dislocation is indicated by the blue line. Note the different rate of step propagation along the vertical axis, relative to the horizontal one, induced by the different energy of unit incorporation in the two directions.

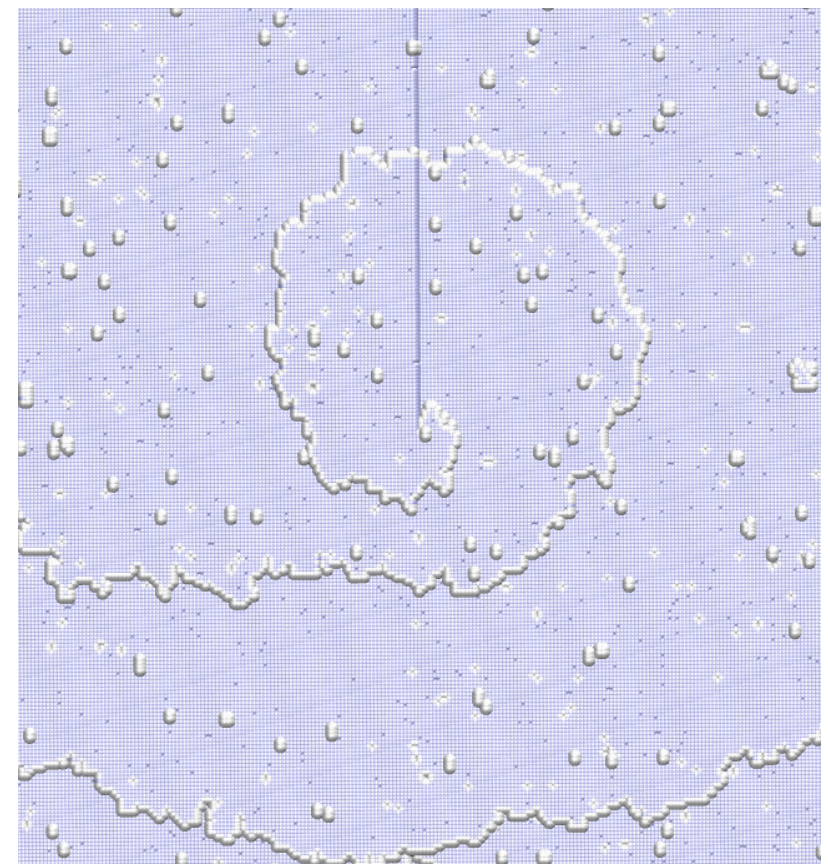


Figure 5. Saturated solution concentration plotted as a function of the particle size. The urea crystals are approximately tetragonal, and therefore the crystal radius has been calculated as the average particle size along the three principal axes.

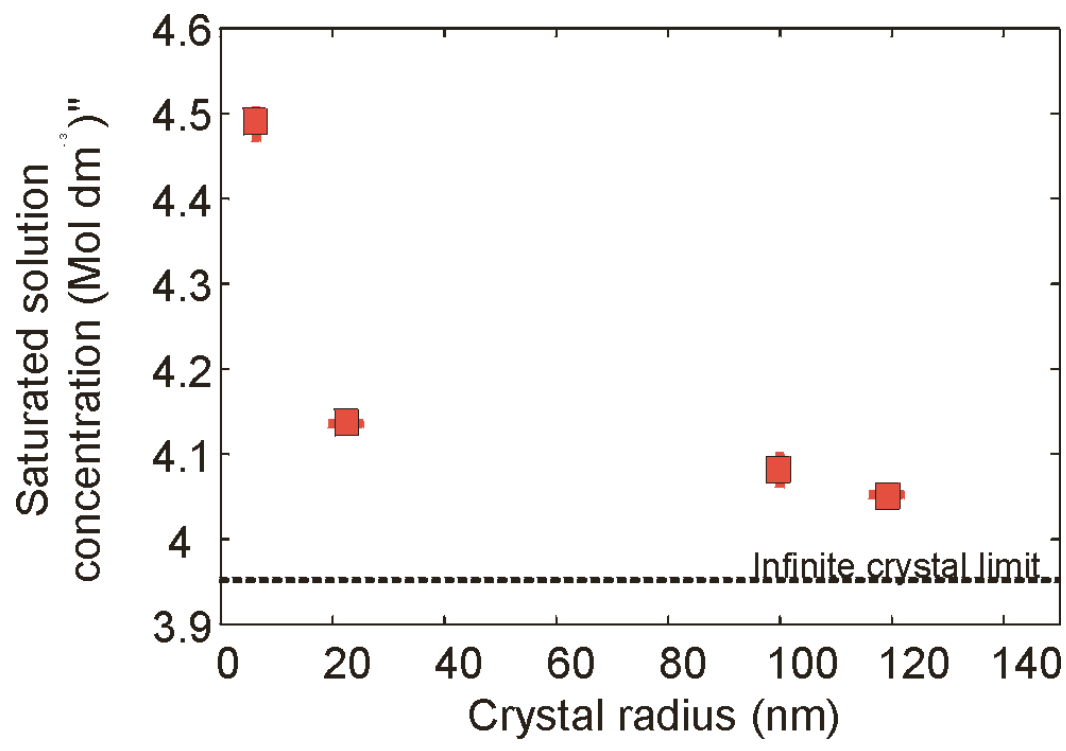

